\title{
Primary pulmonary extranodal NK/T-cell lymphoma: a case report and literature review
}

\author{
Yanli Wang", Zhengxia Wang", Chaojie Wu, Xinyun Zhao, Ningfei Ji, Mao Huang \\ Department of Respiratory and Critical Care Medicine, the First Affiliated Hospital of Nanjing Medical University, Nanjing, China \\ \#These authors contributed equally to this work. \\ Correspondence to: Ningfei Ji; Mao Huang. Department of Respiratory and Critical Care Medicine, the First Affiliated Hospital of Nanjing Medical \\ University, Nanjing 210029, China. Email: jiningfei@163.com, hm6114@163.com.
}

\begin{abstract}
Extranodal natural killer/T-cell lymphoma (ENKTL) is rare lymphoma subtype with a very poor prognosis. ENKTL in the lung is strongly associated with Epstein-Barr virus (EBV) and is extremely rare; only a few cases have been reported. In the present study, we report a case that a 40 -year-old male who presented with cough, sputum and intermittent fever for one month. Chest radiograph revealed progressive multiple nodules in both lungs with ground-glass opacities and bilateral pleural effusion. Based on clinical characteristics and computed tomography (CT) findings, he was initially treated with empirical antibiotics. As there was no significant improvement, bone marrow puncture, left axillary mass biopsy and CT- guided percutaneous lung biopsy were conducted. Therefore, a diagnosis of primary pulmonary ENKTL was confirmed by pathology as cells are positive for CD2, cytoplasmic CD3e, CD56. In situ hybridization for EBV-encoded ribonucleic acid (EBER) was positive. Next generation sequencing (NGS) was used to determine potential therapeutic targets, and the missense mutation of signal transducer and activator of transcription 3 (STAT3) was found. However, the patient demonstrated rapid deterioration and refused chemotherapy. He died shortly following diagnosis. In conclusion: A diagnosis of ENKTL should be considered when patients present with fever and expansive consolidation of the lung, which do not respond to antibiotics. To our knowledge, our patient was the first to undergo NGS for primary pulmonary ENKTL.
\end{abstract}

Keywords: Pulmonary extranodal NK/T-cell lymphoma; EBV-encoded ribonucleic acid; next-generation sequencing; case report

Submitted May 21, 2020. Accepted for publication Sep 26, 2020.

doi: $10.21037 /$ tcr-20-2151

View this article at: http://dx.doi.org/10.21037/tcr-20-2151

\section{Introduction}

Natural killer (NK)/T-cell lymphoma is a rare type of non-Hodgkin's lymphoma with great heterogeneity, high invasive nature and poor prognosis. Extranodal natural killer/T-cell lymphoma (ENKTL) typically involves nasal cavity and upper respiratory tract $(1,2)$. Sometimes, it occurs at extranasal sites such as the skin, gastrointestinal tract, testes, soft tissues and so on $(3,4)$. However, only a few cases of ENKTL in the lungs have been reported. The annual incidence of ENKTL varies by ethnicity and geographical. It is higher in eastern Asia and Latin America compared with European (5). It also has a predominance in men $(6,7)$. Primary pulmonary ENKTL lacks specific clinical manifestations, thus it is usually misdiagnosed in the early stage. In the present study, we report on a case of primary pulmonary ENKTL presenting with pulmonary symptoms and the results of NGS performed in the patient's lung tissue.

We present the following article in accordance with the CARE reporting checklist (available at http://dx.doi. org/10.21037/tcr-20-2151).

\section{Case presentation}

A 40-year-old male was admitted to the Respiratory Department of The First Affiliated Hospital of Nanjing 
Table 1 Laboratory assessments

\begin{tabular}{|c|c|c|}
\hline Variables & Results & Reference range \\
\hline White blood cell count $\left(\times 10^{9} / \mathrm{L}\right)$ & 2.62 & $4-10$ \\
\hline Absolute neutrophil count $\left(\times 10^{9} / \mathrm{L}\right)$ & 2.0 & $2-7.7$ \\
\hline Hemoglobin (g/L) & 123 & $110-160$ \\
\hline Platelet count $\left(\times 10^{9} / L\right)$ & 134 & $100-300$ \\
\hline Alanine aminotransferase (U/L) & 344.5 & $7-40$ \\
\hline Aspartate aminotransferase (U/L) & 273.6 & $13-35$ \\
\hline Albumin (g/L) & 23.5 & $40-55$ \\
\hline Globulin (g/L) & 19.5 & $20-40$ \\
\hline Serum total bilirubin $(\mu \mathrm{mol} / \mathrm{L})$ & 7.7 & $5.1-19$ \\
\hline Direct bilirubin ( $\mu \mathrm{mol} / \mathrm{L})$ & 3.3 & $0-6.8$ \\
\hline Indirect bilirubin $(\mu \mathrm{mol} / \mathrm{L})$ & 4.4 & $0-20$ \\
\hline Lactate dehydrogenase (IU/L) & 1,095 & $140-271$ \\
\hline Urea nitrogen (mmol/L) & 3.2 & $2.9-8.2$ \\
\hline Serum creatinine $(\mu \mathrm{mol} / \mathrm{L})$ & 52.4 & $44-133$ \\
\hline C-reactive protein $(\mathrm{mg} / \mathrm{L})$ & 23 & $0-8$ \\
\hline Erythrocyte sedimentation rate $(\mathrm{mm} / \mathrm{h})$ & 10 & $0-20$ \\
\hline Procalcitonin (ng/mL) & $0.43 \mathrm{~L}$ & $0-0.5$ \\
\hline CEA (ng/mL) & 2.25 & $0-4.7$ \\
\hline AFP (ng/mL) & 0.61 & $0-20$ \\
\hline CA19-9 (U/mL) & 19.57 & $0-39$ \\
\hline Cyfra21-1 (ng/mL) & 4.45 & $0-3.3$ \\
\hline CA72-4 (U/mL) & 1.72 & $0-6.9$ \\
\hline NSE (ng/mL) & $29.75 \mathrm{~L}$ & $0-16.3$ \\
\hline
\end{tabular}

medical university (Nanjing, Jiangsu, China) on May 9, 2018, with cough, sputum and intermittent fever (from $38.5^{\circ} \mathrm{C}$ to a maximum of $39.5^{\circ} \mathrm{C}$ ) for one month. The patient had a 20 -year smoking history and smoked 20 cigarettes daily. There was no relevant personal or familial medical history. The patient was immuno $\neg$ competent and HIV negative.

\section{Physical examination}

On admission, the patient's body temperature was $37.7^{\circ} \mathrm{C}$, pulse rate was 96 b.p.m, respiratory rate was 18 breaths/min and blood pressure $116 / 73 \mathrm{mmHg}$. On chest auscultation, no moist rales were found in both lung fields. No cervical or axillary lymph node enlargement was detected.

\section{Consensus process}

The patient initially received empirical antibiotic treatment with cefdinir. As he showed little response to the therapy, computed tomography (CT) of the chest was performed for diagnosis. Laboratory assessments were conducted. The patient was treated with broad-spectrum antibiotic therapy, but had no relief. Bone marrow puncture and B-mode ultrasound-guided left thoracic puncture were performed. A CT-guided transthoracic needle biopsy of the upper right lung was therefore undertaken. NGS of the tissues was obtained.

\section{Laboratory assessments}

Laboratory assessments were conducted and revealed in Table 1. The artery blood analysis showed $\mathrm{pH} 7.42, \mathrm{PaO}_{2}$ $54 \mathrm{mmHg}, \mathrm{PaCO}_{2} 33 \mathrm{mmHg}, \mathrm{HCO}_{3}^{-}$concentration $24.6 \mathrm{mmol} / \mathrm{L}$, Lac $0.7 \mathrm{mmol} / \mathrm{L}, \mathrm{SaO}_{2}$ concentration $90 \%$. $\mathrm{G}$ test and GM test were both negative, T-SPOT is also negative. Cryptococcus capsular antigen and Legionella antibodies were negative. EBV DNA quantitative was $5.79 \times 10^{3}$ copy $/ \mathrm{mL}$, higher than reference range. No acidfast positive bacilli were found in sputum smear.

On admission, a repeat chest CT revealed progressive multiple nodules in both lungs with ground-glass opacities and bilateral pleural effusion, as shown in Figure 1. Chest and abdominal CT also revealed axillary lymphadenopathy and multiple enlarged lymph nodes around abdominal aorta. These findings suggested an advanced lung cancer or pulmonary infiltration of malignant hematological tumor or severe pneumonia. The patient was then treated with broad-spectrum antibiotic therapy (biapenem, moxifloxacin and voriconazole) for 5 days.

Despite enhanced antibiotic therapy, the patient showed aggravated dyspnea and her clinical condition continued to deteriorate. Meanwhile, the counts of WBC decreased. Both sputum culture and blood culture were negative. Bone marrow puncture results showed significant granulocyte, erythroid and megakaryocyte proliferation, as well as platelet scattering, cell morphology is generally normal (Figure 2A,B,C). The anti-infection treatment was changed to linezolid, voriconazole, and sulfamethoxazole; however, this was unsuccessful. Because of pleural effusion, we performed B-mode ultrasound-guided left thoracic puncture. The pleural effusion routine and biochemical 

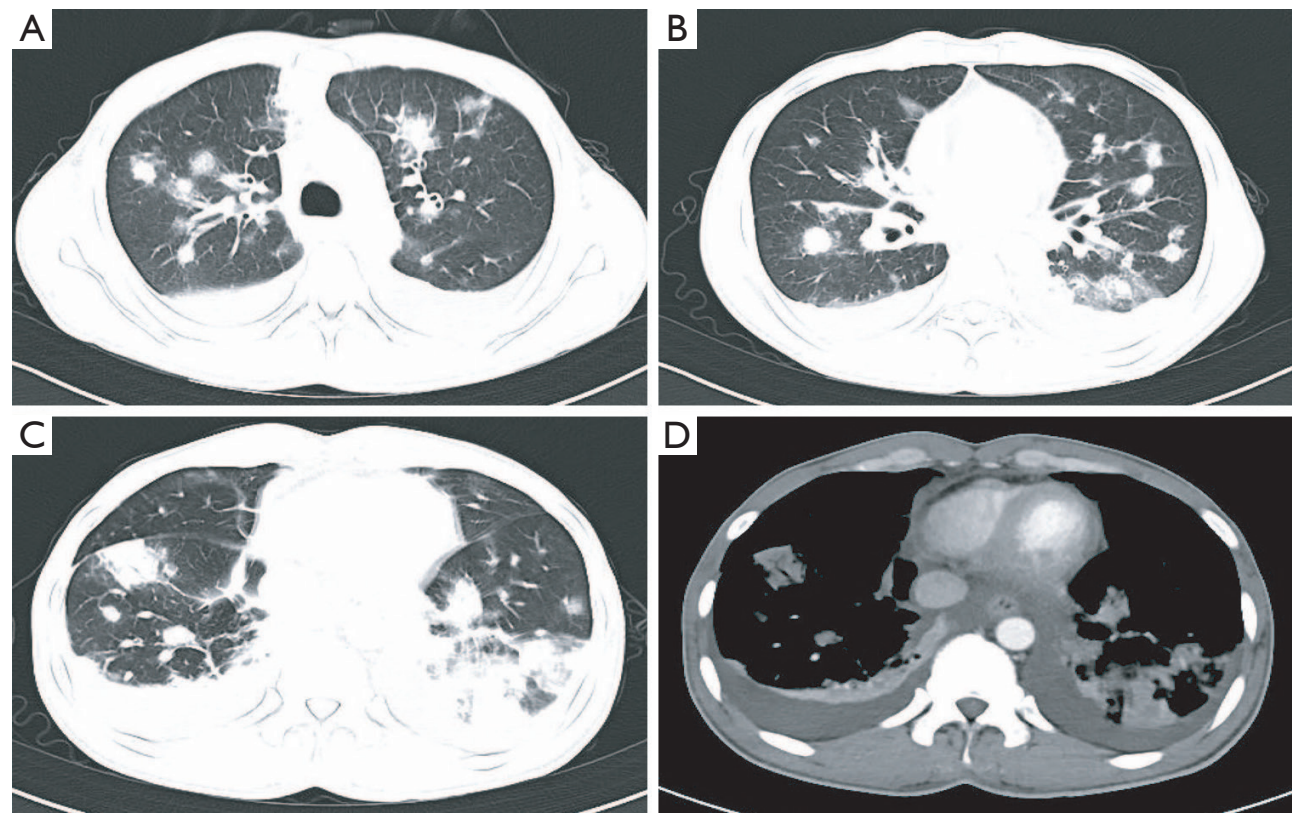

Figure 1 Computed tomography images showing radiological changes. (A-C) The lung window photography of CT showed multiple nodules with ground-glass opacities and consolidation in bilateral pulmonary, thickening of bronchial walls, and interstitial pulmonary edema. (D) The mediastinal window photography of CT showed Mediastinal lymphadenopathy in the pretracheal retrocaval region and pleural effusion accompanied by inadequate expansion of both lower lungs.
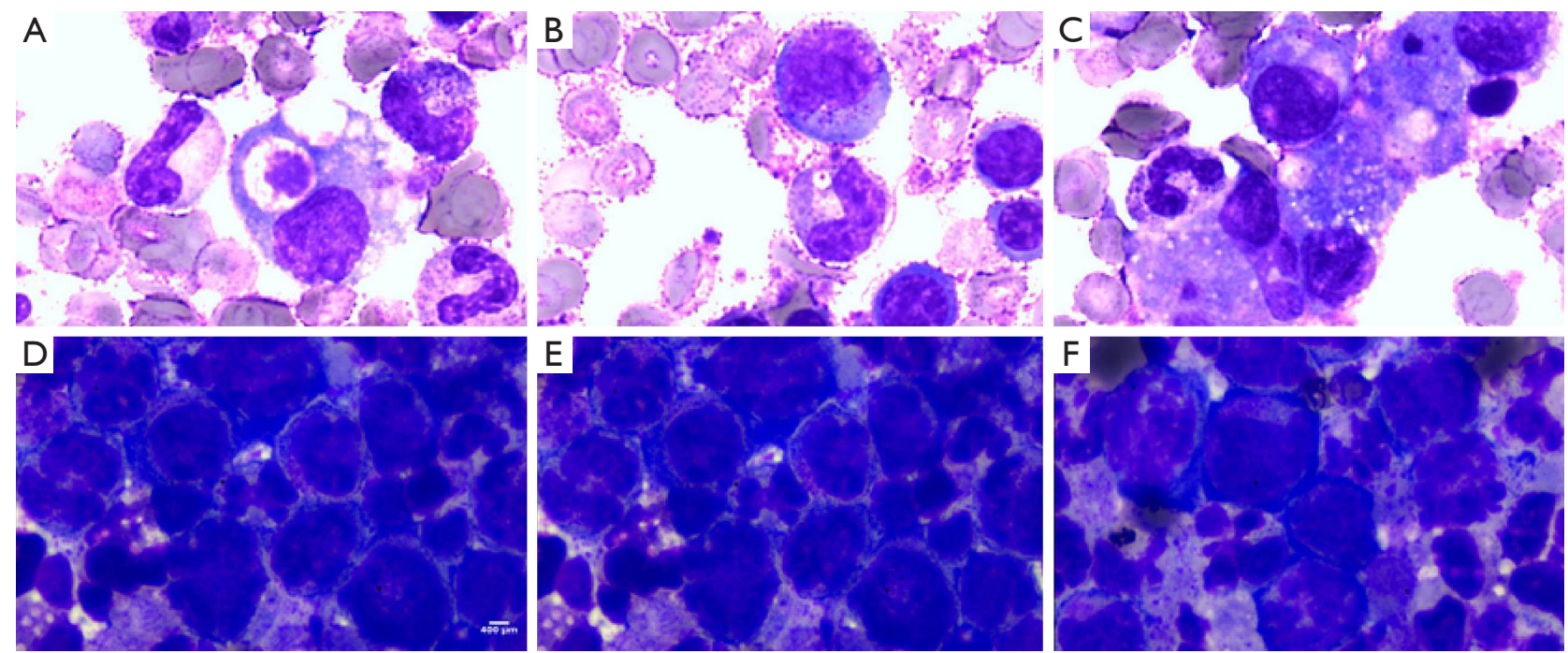

Figure 2 Bone marrow smear and Plasma cell examination in hydrothorax. (A-C) Bone marrow smear with Wright staining showed active proliferation of bone marrow cells, and platelets scattered rarely, cell morphology is generally normal. (D-F) Segmented cells and heteromorphic cells were seen from the cells in hydrothorax with Wright staining (magnification, $\times 50$ ). 

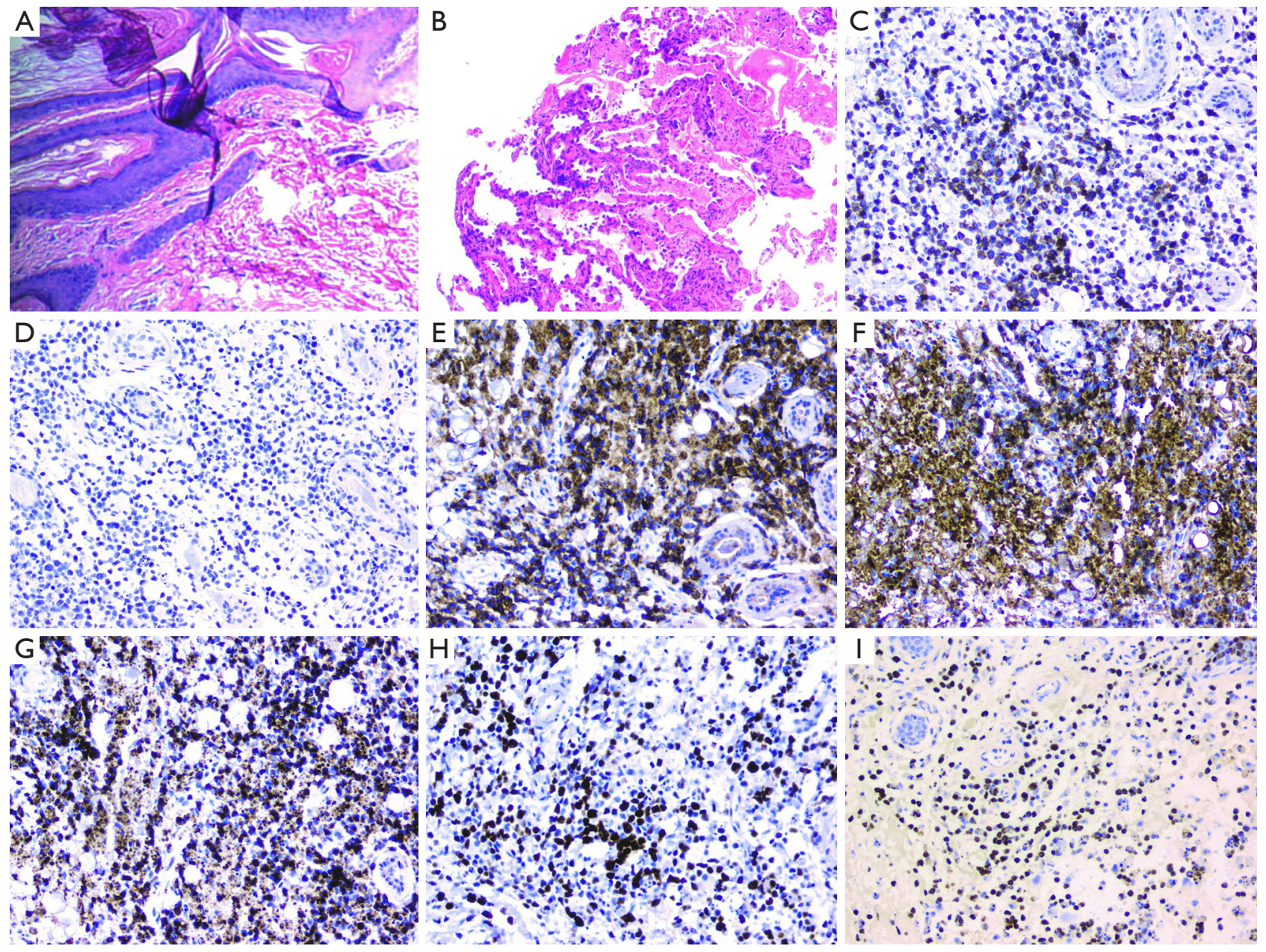

Figure 3 Pathologic findings of Axillary mass and CT-guided transthoracic needle biopsy. (A) Tissue showed Malignant tumor with necrosis, tumor invasion of blood vessel wall and skin accessories, nerve involvement. (B) Percutaneous transthoracic needle biopsy specimen showed chronic inflammation, diffuse alveolar cavity visible large degenerative necrotic exudates and alveolar-epithelial atypical hyperplasia. (C) By immunohistochemistry, cells were positive for CD3. (D) Immunohistochemical staining was negative for CD20. (E) Immunohistochemical staining was positive for CD56. (F-G) Immunohistochemical staining was positive for granzyme B and TIA. (H) cells were positive for Ki67. (I) In situ hybridization for Epstein-Barr virus-encoded RNA (EBER) showed positive reaction in tumor cells (magnification, $\times 100$ ).

results indicated exudate. The CEA in pleural effusion is $5.99 \mathrm{ng} / \mathrm{mL}$, not very high, however, malignant tumor cells were seen in pleural effusion (Figure 2D,E,F). It probably means that the disease has been in advanced stage and has distant metastasis. A mass under the axilla was palpable. The biopsy found a malignant tumor with necrosis, invasion of the vascular wall skin, appendages, and nerve involvement (Figure 3A).

A CT-guided transthoracic needle biopsy of the upper right lung was therefore undertaken. Histologically, the cells presented chronic inflammation, diffuse alveolar cavity visible large degenerative necrotic exudates and alveolar-epithelial atypical hyperplasia (Figure $3 B$ ). Immunohistochemical (IHC) staining was positive for CK7, TTF-1, and CK-pan in alveolar epithelial cells; CD163, CD68 in histocytes; CD31 and CD34for vessels; CD2, LCA,CD56, cytoplasmic CD3 $\varepsilon$ in tissue; and negative for CD20 (Figure 3C,D,E,F,G,H). The antibodies of IHC were purchased from Maxin BIO (Fuzhou, China). The IHC staining was performed according to the manufacturer's instructions. In situ hybridization showed that tumor cells were positive for Epstein-Barr virus encoded RNA (EBER), 
Table 2 Gene mutational profile by next-generation sequencing

\begin{tabular}{llcc}
\hline Gene & Variation type & Variation result & Mutation abundance \\
\hline STAT3 & Missense mutation & NM_139276.2(STAT3):c.1842C>G(p.Ser614Arg) & $27.55 \%$ \\
EP300 & Nonsense mutation & NM_001429.3(EP300):c.4449C>G(p.Tyr1483*) & $20.35 \%$ \\
\hline
\end{tabular}

as shown in Figure 3I. These findings were consistent with NK/T-cell lymphoma. In order to investigate the molecular characteristics of the NK/T-cell lymphoma and to identity new therapeutic strategies, NGS of the tissues obtained at diagnosis was performed at Beijing Genomics institution (Shenzhen, China). Tissue gene tests showed missense mutation of STAT3 as NM_139276.2(STAT3): c. $1842 \mathrm{C}>\mathrm{G}(\mathrm{p}$.Ser614Arg) with abundance $27.55 \%$, as shown in Table 2. The patient was diagnosed with primary pulmonary ENKTL. Unfortunately, the patient suffered rapid deterioration with respiratory failure, hepatic failure and hemophagocytic syndrome. He refused chemotherapy. Then the patient was transferred to ICU, however, he died 3 days later as a result of disease progression.

All procedures performed in studies involving human participants were following the ethical standards of the institutional and/or national research committee(s) and with the Helsinki Declaration (as revised in 2013). Written informed consent was obtained from the patient.

\section{Discussion}

Extranodal ENKTL is a rare non-Hodgkin lymphoma with a highly invasive nature. It has obvious regional and ethnic differences, which is more common in eastern Asia, central and South America, it has a higher predominance among men (7). The primary sites of ENKTL are mainly in the upper respiratory tract, with nasal cavity, sometimes from the skin, testicles, gastrointestinal tract, and so on. As primary pulmonary ENKTL is rare, and because it has nonspecific clinical symptoms and limited availability of biopsy tissues, its diagnosis is difficult. In previously published studies, it has been reported that most patients present with fever, cough, and dyspnea (8). Most patients were initially misdiagnosed as pneumonia and treated with empirical antibiotics (9). If clinical symptoms show deterioration, despite adequate antibiotic therapy, bronchoscopy examination or CT-guided needle biopsy should be conducted (4). ENKTL should also be differentiated from pneumomycosis, tuberculosis and lung cancer (10).

At present, the specific pathogenesis of ENKTL is unclear. It has been reported that the occurrence of ENKTL is closely related to EBV infection, and the copy number of EBV-DNA is positively correlated with disease severity (11). In this case, there was a significant increase in plasma EBV-DNA levels, and IHC results showed positive expression of EBV. It is in accordance with the previous reports that almost all cases were positive for the EBV, proven either by elevated plasma EBV-DNA levels or positive in situ hybridization for EBER (1).

NK/T-cell lymphoma is an aggressive disease with poor response to therapy, resulting in low survival rate. The early correct diagnosis can effectively improve the complete remission rate and 5-year survival rate (12). ENKTL is associated with shorter survival rate and poorer therapy response irrespective of the stage, compared with nasal disease. The aggressive clinical behavior of primary extranasal disease is similar to that of advanced stage nasal disease (13). The diagnosis of ENKTL cell lymphoma mainly depends on biopsy and immunohistochemistry experiments, and the results of immunohistochemistry experiments are the cornerstone of the diagnosis. In the immunohistochemical results of lung biopsy tissue in this case, CD2, CD163, CD68, LCA, especially EBER were positive and CD20 negative, which were similar to the reported results of several cases of typical NK/T-cell lymphoma (14). Therefore, there was sufficient pathological evidence for the diagnosis of primary pulmonary NK/T-cell lymphoma in this case.

As molecular pathology of NK/T-cell lymphoma, the deletion of chromosome $6 \mathrm{q}(6 \mathrm{q}-)$ is considered to be a cytogenetic aberration by conventional karyotyping. Furthermore, gene expression profiling reveals that NK/ T-cell lymphoma is similar to EBV-positive peripheral T-cell lymphoma of $\gamma / \delta$ subtype (6). The underlying mechanism includes JAK/STAT activation, over-expression of aurora kinase A and NK- $\kappa \mathrm{B}(6)$. NGS has provided that there is activating STAT3 and STAT5B mutation in major cases (15), mutation of the RNA helicase gene DDX3X in $20 \%$ cases, and other mutated genes such as TP53, EP300 in a few cases (13). In this case, NGS was performed for the first time in primary pulmonary ENKTL. The missense 
mutation of STAT3 was found, in according with the reports.

There are no large randomized clinical trials comparing the efficacy of different regimens, so there is no standard treatment for primary pulmonary NK/T-cell lymphoma. For early stage ENKTL, a combination of radiotherapy and chemotherapy is considered to be effective for extending the survival time; for advanced stage, chemotherapy remains the main treatment but with poor curative effect because of multidrug resistance (2). Therefore, more effective treatment strategies are required for this disease.

The patient suffered rapid deterioration and refused chemotherapy. There is not much time for treatment.

\section{Conclusions}

In conclusion, primary pulmonary NK/T-cell lymphoma is a systemic disease with highly aggressive behavior and poor prognosis. Early diagnosis is very important to improve the prognosis and prolong the survival time. However, at the early stage, it lacks of specific clinical manifestation and it is easy to be misdiagnosed and missed. Therefore, repeated biopsy is necessary and cytogenetic testing might offer a prospective method for the diagnosis of this disease. The diagnosis should be established by the combination of clinical manifestation, comprehensive imaging, histopathological and molecular examination, and much more attention should be paid to this disease. Furthermore, prospective multicenter studies should be conducted to define the best therapeutic strategies and prognostic factors.

\section{Acknowledgments}

Funding: This work was supported by the National Natural Science Foundation of China grant 81800090 , 81770031, Natural Science Foundation of Jiangsu Province BK20171501, the "Six Talent Peak" Project of Jiangsu Province 2013-WSN-059, a project from the Administration of Traditional Chinese Medicine of Jiangsu Province LZ13213 and a project from Jiangsu Provincial Commission of Health and Family Planning H201501.

\section{Footnote}

Reporting Checklist: The authors have completed the CARE reporting checklist. Available at http://dx.doi.org/10.21037/ tcr-20-2151
Conflicts of Interest: All authors have completed the ICMJE uniform disclosure form (available at http://dx.doi. org/10.21037/tcr-20-2151). The authors have no other conflicts of interest to declare.

Ethical Statement: The authors are accountable for all aspects of the work in ensuring that questions related to the accuracy or integrity of any part of the work are appropriately investigated and resolved. All procedures performed in studies involving human participants were in accordance with the ethical standards of the institutional and/or national research committee(s) and with the Helsinki Declaration (as revised in 2013). Written informed consent was obtained from the patient.

Open Access Statement: This is an Open Access article distributed in accordance with the Creative Commons Attribution-NonCommercial-NoDerivs 4.0 International License (CC BY-NC-ND 4.0), which permits the noncommercial replication and distribution of the article with the strict proviso that no changes or edits are made and the original work is properly cited (including links to both the formal publication through the relevant DOI and the license). See: https://creativecommons.org/licenses/by-nc-nd/4.0/.

\section{References}

1. Chien CC, Lee HS, Lin MH, et al. Primary extranodal natural killer/T-cell lymphoma of bronchus and lung: A case report and review of literature. Thorac Cancer 2016;7:140-4.

2. Ding W, Wang J, Zhao S, et al. Clinicopathological study of pulmonary extranodal nature killer/T-cell lymphoma, nasal type and literature review. Pathol Res Pract 2015;211:544-9.

3. Du J, Ma PP, Wang QY, et al. Extranodal NK/T-cell lymphoma, nasal type: a case report of 7-year natural course and review of literature. Int J Clin Exp Pathol 2015;8:9620-8.

4. Zhang J, Wang $M$, Yang $X$, et al. Primary pulmonary extranodal NK/T-cell lymphoma of nasal type misdiagnosed as pneumonia: A case report and literature review. Medicine (Baltimore) 2017;96:e8914.

5. Bassig BA, Au WY, Mang O, et al. Subtype-specific incidence rates of lymphoid malignancies in Hong Kong compared to the United States, 2001-2010. Cancer Epidemiol 2016;42:15-23.

6. Lin GW, Xu C, Chen K, et al. Genetic risk of extranodal 
natural killer T-cell lymphoma: a genome-wide association study in multiple populations. Lancet Oncol 2020;21:306-16.

7. Perry AM, Diebold J, Nathwani BN, et al. Non-Hodgkin lymphoma in the developing world: review of 4539 cases from the International Non-Hodgkin Lymphoma Classification Project. Haematologica 2016;101:1244-50.

8. Qiu Y, Hou J, Hao D, et al. Primary pulmonary NK/T-cell lymphoma: A case report and literature review. Mol Clin Oncol 2018;8:753-6.

9. Pan Z, Xu ML. T-cell and NK-cell lymphomas in the lung. Semin Diagn Pathol 2020;37:273-82.

10. Hu Q, Xu L, Zhang X, et al. Primary pulmonary extranodal natural killer/T-cell lymphoma (ENKTL), nasal type: Two case reports and literature review. Medicine (Baltimore) 2020;99:e20822.

Cite this article as: Wang $\mathrm{Y}$, Wang $\mathrm{Z}, \mathrm{Wu} \mathrm{C}, \mathrm{Zhao} \mathrm{X}$, Ji N, Huang M. Primary pulmonary extranodal NK/T-cell lymphoma: a case report and literature review. Transl Cancer Res 2020;9(11):7359-7365. doi: 10.21037/tcr-20-2151
11. Kidwai SM, Parasher AK, Lin FY. An unusual presentation of NK/T-cell lymphoma, nasal-type in the United States. Am J Otolaryngol 2015;36:80-3.

12. Chauchet A, Michallet AS, Berger F, et al. Complete remission after first-line radio-chemotherapy as predictor of survival in extranodal NK/T cell lymphoma. J Hematol Oncol 2012;5:27.

13. Tse E, Kwong YL. NK/T-cell lymphomas. Best Pract Res Clin Haematol 2019;32:253-61.

14. Jabbari Azad F, Delavarian Z, Hatami M, et al. Extranodal NK/T Cell Lymphoma with Destruction of the Uvulae: A Case Report. Iran J Otorhinolaryngol 2017;29:101-8.

15. Küçük C, Jiang B, Hu X, et al. Activating mutations of STAT5B and STAT3 in lymphomas derived from $\gamma \delta$-T or NK cells. Nat Commun 2015;6:6025. 